\title{
Does The Temperature Of A Black-Body Depend On The Frame Of Reference?
}

\section{A. Dutta Roy}

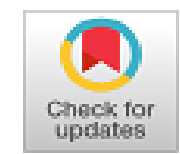

\begin{abstract}
In this manuscript, we establish a mathematical model to determine whether the thermodynamic temperature of a black-body depends on the Inertial Frame of Reference as viewed by different observers situated in diffserent Inertial Frames or whether the temperature of a black-body does not depend on the Inertial Frame of Reference. Stefan - Boltzmann Law is used to establish the mathematical model and finally the conclusion/s are drawn.
\end{abstract}

Keywords : Black-Body, Inertial Frame of Reference, StefanBoltzmann Law, Temperature.

\section{INTRODUCTION}

Introduction of Special Theory of Relativity led to the question - Does temperature of a body depend on its frame of reference? This subject has been researched by Planck, Ott, Avramov [1], Bormashenko [2] et al. However, there still does not exist a consensus related to the subject. Considering, the works of all the above-mentioned researchers, the author has tried to establish mathematically, whether temperature of a body is frame-dependent or frameindependent. Section I (INTRODUCTION) gives us an introduction to the topic that is going to be investigated. Section II (RELATED WORK IN THIS FIELD) tells about work done by other authors related to this topic. Section III (METHODOLOGY AND DERIVATIONS) is the main section where mathematics and reasoning are used to reach a conclusion regarding the topic. Section IV (RESULT ANALYSIS) is about interpreting the results obtained in Section III. Section V (CONCLUSION AND FUTURE WORK) deals with the conclusion. A Reference Section, at the last, provides relevant references.

\section{RELATED WORK IN THIS FIELD}

After the publication of Special Theory of Relativity, Planck studied the effects of relativity on thermodynamic properties of a body. Planck concluded that temperature must be Lorentz co-variant [3]. Therefore, it was stated:

Revised Manuscript Received on October 30, 2019.

* Correspondence Author

A. Dutta Roy*, Independent Researcher, Kolkata, India.

(c) The Authors. Published by Blue Eyes Intelligence Engineering and Sciences Publication (BEIESP). This is an open access article under the CC BY-NC-ND license (http://creativecommons.org/licenses/by-nc-nd/4.0/)
$T^{\prime}=T \sqrt{1-\frac{v^{2}}{c^{2}}}$ where $\mathrm{T}^{\prime}$ is the temperature observed by the inertial frame of reference moving with a constant velocity $\mathrm{v}$ while $\mathrm{T}$ is the temperature observed by the inertial frame of reference at rest. However, in 1963, Ott deduced a different equation which can be written as follows [4]:

$T^{\prime}=T \frac{1}{\sqrt{1-\frac{v^{2}}{c^{2}}}}$ following which different authors have given their own opinion on the subject.

\section{METHODOLOGY AND DERIVATIONS}

Let a system of co-ordinates $(\mathrm{x}, \mathrm{y}, \mathrm{z}, \mathrm{t})$ possess a flat square shaped black body, placed along the $\mathrm{X}-\mathrm{Z}$ plane whose temperature is measured as $\mathrm{T}$ in the above-mentioned system. The black body releases electro-magnetic radiation. Let this flat black body send out, in the direction of $\mathrm{x}$-axis (in a direction parallel to $\mathrm{x}$-axis) plane waves of electromagnetic radiation containing energy $\mathrm{L}$ in one direction. Similarly, the other face of the black body sends out energy $\mathrm{L}$ in the opposite direction. These energies are measured in the system $(x, y, z, t)$. Further, the flat black body is at rest in the system $(x, y, z, t)$. Let this frame of reference be referred as frame 1 . Now, a new system of co-ordinates is introduced. This new system of co-ordinates $\left(x^{\prime}, y^{\prime}, z^{\prime}, t^{\prime}\right)$ is moving in uniform parallel translation with respect to frame 1 and has its origin of co-ordinates in motion along the axis of $\mathrm{x}$, with a velocity $\mathrm{v}$. Let this frame of reference be referred as frame 2. Using relativistic Doppler effect, the energy of electromagnetic radiation, released by the black body in frame 2 will be seen/ calculated as follows [5]:

$L_{1}=L \frac{1-\frac{v}{c}}{\sqrt{1-\frac{v^{2}}{c^{2}}}}$
$L_{2}=L \frac{1+\frac{v}{c}}{\sqrt{1-\frac{v^{2}}{c^{2}}}}$

Therefore, energy released by the black body in frame 1 is $\mathrm{L}$ $+\mathrm{L}=2 \mathrm{~L}$. While energy released by the black body as measured by frame 2 is as follows: 


$$
\begin{aligned}
& L_{1}+L_{2}=L \frac{2}{\sqrt{1-\frac{v^{2}}{c^{2}}}} \\
& \frac{\text { Energy released as calculated by frame } 2}{\text { Energy released as calculated by frame } 1}=\frac{1}{\sqrt{1-\frac{v^{2}}{c^{2}}}}
\end{aligned}
$$

To find Energy released by the black body in frame 1, we will use the Stefan - Boltzmann law and the calculation is done as follows:

$j^{*}=\sigma T^{4}$

(5) where $j^{*}$ is the black body's radiant emittance which is directly proportional to the fourth power of the black body's thermodynamic temperature $T$. The constant of proportionality, $\sigma$ is called the Stefan - Boltzmann constant. From Equation (5), the total power radiated by the black body is calculated as follows:

$P=A j^{*}$

where, $\mathrm{A}$ is the total surface area of the black body. (Since, we considered a flat square shaped black body if the length of the edge is $l$ units then total surface area would be $2 l^{2}$ ). Let us consider, an interval of time $t$ in frame 1 . In this interval of time, the total energy radiated by the black body would be calculated as follows:

$E_{1}=P X t$

$E_{1}=A j^{*} X t$

$E_{1}=A X \sigma T^{4} X t=A \sigma T^{4} t$

where $E_{1}$ is the total energy radiated by the black body as calculated by frame 1 through an interval of time t. However, earlier we assumed that the energy released by the black body in frame 1 is $2 \mathrm{~L}$. Therefore, we can say that:

$2 L=A \sigma T^{4} t$

Now, let us look at the black body energy radiation as observed by frame 2. First, let us state one of the important postulates of Special Theory of Relativity [6]:

The laws by which the states of physical systems alter are independent of the alternative, to which of two systems of coordinates, in uniform motion of parallel translation relatively to each other, these alterations of state are referred.

Therefore, Stefan - Boltzmann law in frame 2 will have the following form:

$j^{* \prime}=\sigma^{\prime}\left(T^{\prime}\right)^{4}$

where, $j^{* \prime}$ is the radiant emittance of the black body which resides in frame 1 as observed by frame 2. $T^{\prime}$ is the thermodynamic temperature of the black body as observed by frame 2. $\sigma^{\prime}$ is the constant of proportionality between the radiant emittance as observed by frame 2 and the thermodynamic temperature of the black body as observed by frame 2 .

$\pi$ is a constant of proportionality between area and radius of a circle. The value of $\pi$ remains the same in all inertial frames. It can be logically said that constants of proportionality between two quantities remain the same across all inertial frames. Therefore, we can conclude the following:

$\sigma$

(10)

The total power radiated by the black body as calculated from frame 2 is as follows:
$P^{\prime}=A^{\prime} j^{*^{\prime}}$

(11) where $A^{\prime}$ is the total surface area of the black body as observed from frame 2 .

The black body is placed along the $\mathrm{X}-\mathrm{Z}$ plane in frame 1 . Frame 2 is moving in uniform parallel translation with respect to frame 1 and has it's origin of co-ordinates in motion along the asxis of $\mathrm{x}$, with a velocity $\mathrm{v}$. Thus, the length and breadth of the black body is observed to be the same from frame 2 as observed in frame 1 . Therefore, the following can be stated:

$A^{\prime}=A$

Using Equation (9), (10) and (12), Equation (11) can be written as follows:

$P^{\prime}=A \sigma\left(T^{\prime}\right)^{4}$

(13)

The interval of time $t$ in frame 1 would be calculated as $t$ ' in frame 2. From frame 2, it is observed that frame 1 is moving with a velocity of $v$. Therefore, relation between $t$ ' and $t$ can be written as:

$t^{\prime}=\gamma t$

(14) where $\gamma$ is the Lorentz Factor. Therefore, the total energy radiated by the black body as calculated from frame 2 would be as follows:

$E_{2}=P^{\prime} X t^{\prime}$

$E_{2}=A \sigma\left(T^{\prime}\right)^{4} t^{\prime}$

(15)

From Equation (4), discussed above, we concluded that:

$\frac{E_{2}}{E_{1}}=\frac{1}{\sqrt{1-\frac{v^{2}}{c^{2}}}}=\gamma$

(16)

Putting Equation (15) and Equation (7) in Equation (16) it can be seen:

$\frac{E_{2}}{E_{1}}=\gamma$

$\frac{A \sigma\left(T^{\prime}\right)^{4} t^{\prime}}{A \sigma T^{4} t}=\gamma$

$A \sigma\left(T^{\prime}\right)^{4} t^{\prime}=\gamma A \sigma T^{4} t$

Cancelling the common factors and using Equation (14) we get the following:

$\left(T^{\prime}\right)^{4} \gamma t=\gamma T^{4} t$

Further, cancelling the common factors, the following is obtained:

$\left(T^{\prime}\right)^{4}=T^{4}$

(17)

Taking the fourth root and considering only positive value for absolute temperature it can finally be written:

$T^{\prime}=T$

\section{RESULT ANALYSIS}

From our mathematical equations and logical reasoning in Section III, we came to the final equation which is stated as follows:

$T^{\prime}=T$

(19) $\mathrm{T}$ is the thermodynamic temperature of the black body as measured from frame 1 and $\mathrm{T}^{\prime}$ is the thermodynamic temperature of the same black body as measured from frame 2. 
Analysis

The results obtained shows that the thermodynamic Temperature of a Black-Body does not depend on the Frame of Reference.

\section{CONCLUSION AND FUTURE WORK}

The main conclusion of the manuscript is thermodynamic Temperature of a Black-Body does not depend on the Frame of Reference. For future scope of this topic, the author will try to prove the same equation using the Ideal Gas Equation.

\section{REFERENCES}

1. I. Avramov, Relativity and Temperature, Russian Journal of Physical Chemistry, vol. 77, suppl. 1,2003,pp S179-S182.

2. E. Bormashenko, Entropy of Relativistic Mono-Atomic Gas and Temperature Relativistic Transformation in Thermodynamics, Entropy, 2007, 9, 113-117.

3. M. Planck, Ann.Phys.Leipzig, 1908, vol. 26

4. H. Ott, Lorenz transformation der Warme und der Temperatur, Z. Phys., 1963, vol. 175, no 1

5. A. Einstein, Does the Inertia of a Body Depend upon its EnergyContent?, Annalen der Physik. 18:639, 1905

6. A. Einstein, Does the Inertia of a Body Depend upon its EnergyContent?, Annalen der Physik. 18:639, 1905

\section{AUTHORS PROFILE}

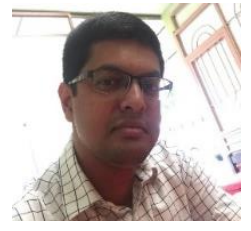

A. Dutta Roy holds a Bachelor of Engineering degree. $\mathrm{He}$ is an Independent Researcher with interest in all forms of Theoretical Physics and wants to understand the workings of the Universe from the Quantum scale to Astronomical scale. His plans include obtaining master's and $\mathrm{PhD}$ and pursuing full-time research. 